\title{
The Design of Fall Detection and Positioning Alarm System Base on WCDMA
}

\author{
Guangmin Sun, Meng Zhang and Xu Yao \\ School of Electronic Information and Control Engineering, Beijing University of Technology, Beijing, China \\ gmsun@bjut.edu.cn
}

\begin{abstract}
In this paper, a novel fall detection and automatic alarm system has been designed and presented, which consists of sensor module, control module and communication module. The sensor modules composed of 3-axis accelerometers are placed on object's pocket to monitor the object's acceleration. System is driven by an inexpensive and low consumption microcontroller. When object is fall, the system will detect it by the abnormal acceleration and make accurate positioning through GPS immediately. The alarm as well as location information is sent via $3 \mathrm{G}$ network to the mobile phone number that has been set. The system has the advantages of portable, low-power and accuracy.
\end{abstract}

Index Terms - Fall Detection, Positioning, WCDMA

\section{Introduction}

In recent years, population aging is a highly generalized process. It is most advanced in the most highly developed cities. According to statistics of Chinese population, elderly population aged 65 and above from the 62.99 million of 1990 to 88.11 million of 2000 , and the total population increased from $5.57 \%$ to $6.96 \%$. In 2040, the proportion of aged 65 and above in the total population will exceed $20 \%$ [1]. Population aging brings about many problems. Nurses are scanty and expenditure of health-nursing is increased. Therefore, carrying out electronic health to improve the independence of elderly people in unsupervised living environments is necessary.

Fall become a major causes of the elder' accidental injury. Of the total population of elders above 65 years of age, 30 percent of the older persons fall at least once a year [2]. If fall not be detected and rescued promptly, the injury will aggravate, even threatening life. Hence an automatic fall detection and alarm device is developed so that the elderly live securely in a separate environment.

Recently, many methods have been proposed to detect fall events. A summary of the state of the art is shown in Table I. Most of the research groups use accelerometers integrated into small boxes worn [3-4] [6-7]. Reference [5] use eight pairs of force sensing resistors integrated into shoes. From the above, analyzing acceleration information to detect fall events is one of the most convenient and simple methods. Reference [7] design a fall detection algorithm based on a tri-axial accelerometer through calculating the value of Sa (sum-vector of three axes) and detection of an exceeding of predefined thresholds. In [6], they also use this algorithm and implement a system integrate with a real-time fall detection band, a home server, and GSM instant messaging function. So we still use $\mathrm{Sa}$, an important and obvious character of activities, as the judgment standards.
However, existing achievements failed to find the location of falling, which increase the difficulty to find where the falling elder especially outdoor activity lead to lost the best opportunity to rescue them. The use of the home server will increase the cost of the system and discommode the users. Therefore, we design a novel fall detection and automatic alarm system, which implement the function of fall detection, accurate location and alarm timely.

In this paper, we integrate all modules in a device with extract data, signal processing, fall detection and alarm without the help of other device, which greatly increase system's portability and reduce system cost. We first proposed integrated WCDMA module into the system, which can make accurate positioning rapidly by GPS and send messages to the pre-set phone number automatically once detected fall.

TABLE I Summary of the State of The Art

\begin{tabular}{|c|l|c|c|c|}
\hline Ref. & Sensor & Location & Detection & Alarming \\
\hline$[3]$ & accelerometer & chest & PDA & PDA \\
\hline$[4]$ & accelerometer & waist & onboard & CMDA \\
\hline$[5]$ & force sensing resistors & shoe & onboard & Not mentioned \\
\hline$[6]$ & accelerometer & waist & Home server & GSM \\
\hline$[7]$ & accelerometer & head & PC & Not mentioned \\
\hline
\end{tabular}

\section{Sensing Modules and Method of Detection}

A. Data Extraction by Tri-axis Accelerometers

In the system, raw data are extracted by a tri-axis accelerometers, LIS331DLH from STMicroElectronics, Inc., which can provide 12 bit acceleration data (i.e. raw data) for each orthogonal axis (ax, ay, az of accelerometer), as shown in Fig.1. The accelerometer can be used to measure both dynamic acceleration (e.g. vibration and acceleration due to movements in general) and static acceleration (e.g., gravity). In steadystate conditions, the net force sensed by accelerometer is gravity only. 


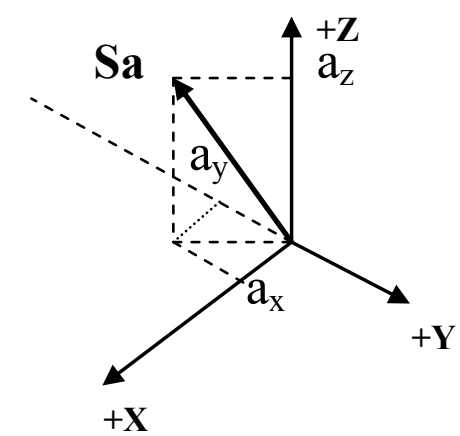

Fig. 1 Coordinate system of accelerometer.

\section{B. Sum-vector of Three Axes}

Due to the sum-vector of three axes freeing from the influence of the position and angle where user wear, we use Sa as the only judgment standards. The extracted tri-axial acceleration value was input to ADuC7020 to compute its $\mathrm{Sa}$, as in equation (1). Sa is used to describe the variation of acceleration during the falling interval [5], and it is a constant value $(1 \mathrm{~g})$ when the body keeps still. Sa is less than $1.5 \mathrm{~g}$ in normal activities of daily living; but while body is in falls, $\mathrm{Sa}$ will exceed the threshold for an extended period of time.

$$
S a=\sqrt{a_{x}^{2}+a_{y}^{2}+a_{z}^{2}} .
$$

\section{Process of Fall Recognition}

In the ordinary walking process for the elderly people, the variation of acceleration is not too great due to the slow movements of elderly ones. The most obvious change of acceleration is in the action of sitting down, as shown in Fig. 2.

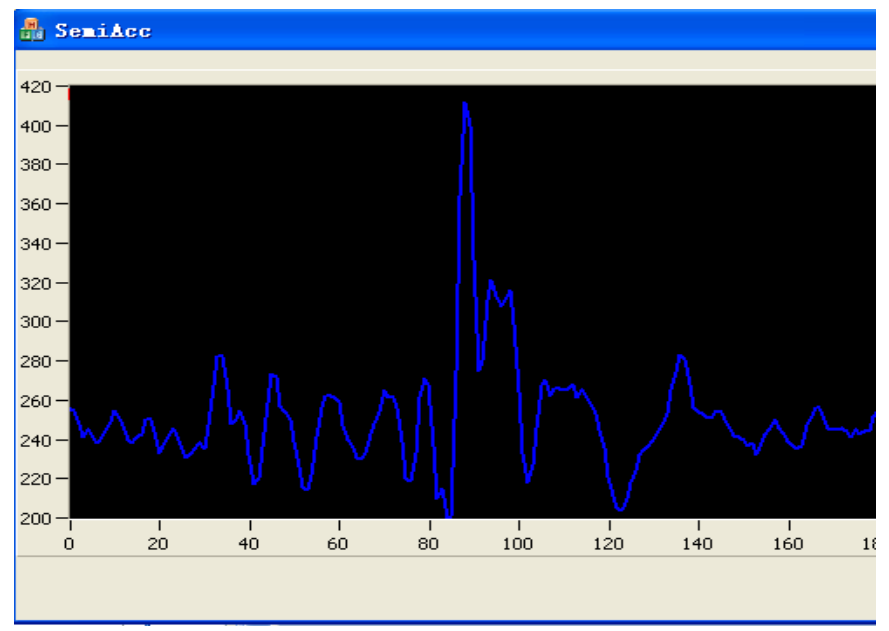

Fig. 2 The variation curve of Sa in the process of sitting down.

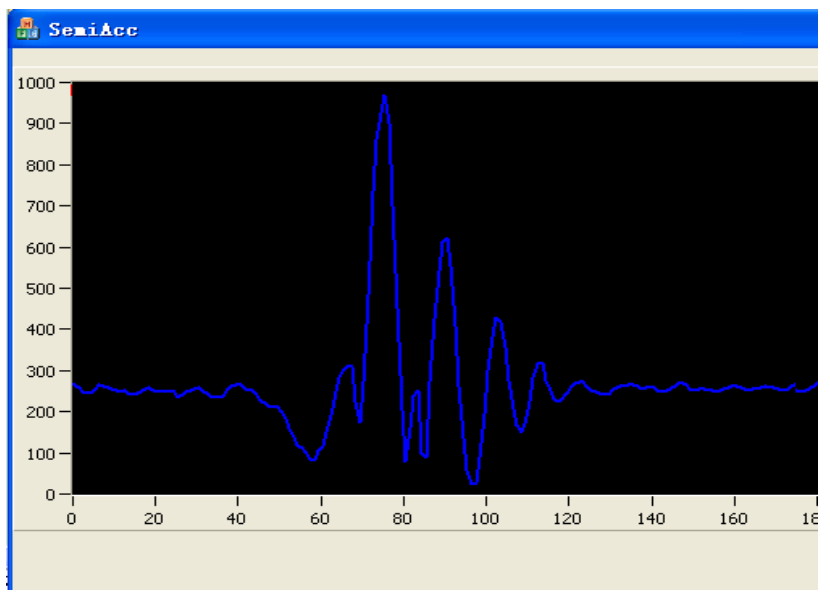

Fig. 3 The variation curve of Sa in the process of accidental fall.

Fig. 3 shows that the variation curve of $\mathrm{Sa}$ in the process of accidental fall. Through the comparison of Fig. 3 and Fig. 2, we can find that the change of acceleration in the fall process has three main features, which can be used as the fall detection criteria.

Certain weight loss phenomenon will occur in the beginning of the fall and the sum-vector of acceleration will be reduced to close to $0 \mathrm{~g}$. For the general fall, weightlessness is not so obvious like free fall, but the Sa will less than $1 \mathrm{~g}$ ( $\mathrm{Sa}$ should be greater than $1 \mathrm{~g}$ in the normal state). After the transient weightlessness, the body will have a collision with the ground. This process will make a great impulse in the curve of Sa. In general, elder can't stand up immediately after a fall, so there is a period of stationary state.

Threshold is set by the characteristics of the above three stages of fall, as the judgment standard, which constitute the entire fall detection algorithm. In reference [3], they consider a fall occurred once at least 10 sampling points are detected in $4 \mathrm{~s}$, but it can't meet the requirements of modern medical detection. So we take $100 \mathrm{~Hz}$ as sampling frequency.

As shown in Fig. 3, the $\mathrm{Y}$-axis of the coordinate represented the value of $\mathrm{Sa}(256 / 1 \mathrm{~g})$. The $\mathrm{X}$-axis represented time intervals, with 100 data entries per second. The experimental data shows that when a fall occurred, the immediate Sa value reduced to $0.8 \mathrm{~g}$ and then it exceeded $3.5 \mathrm{~g}$.

\section{Communication Module and Alarm Function}

\section{A. WCDMA Module}

In global three major $3 \mathrm{G}$ standards, WCDMA is the most mainstream standards with high technology maturity and complete industrial chain, which can realize the global wide range of roaming. SIMCom Company launched a WCDMA module with high performance-price ratio, SIM5320. The module is a castle-style SMT package with a compact size, ultra-thin thickness, and built-in GPS, which is an ideal module for the system designed in this paper and can achieve the function we required. The module is shown in Fig. 4. 


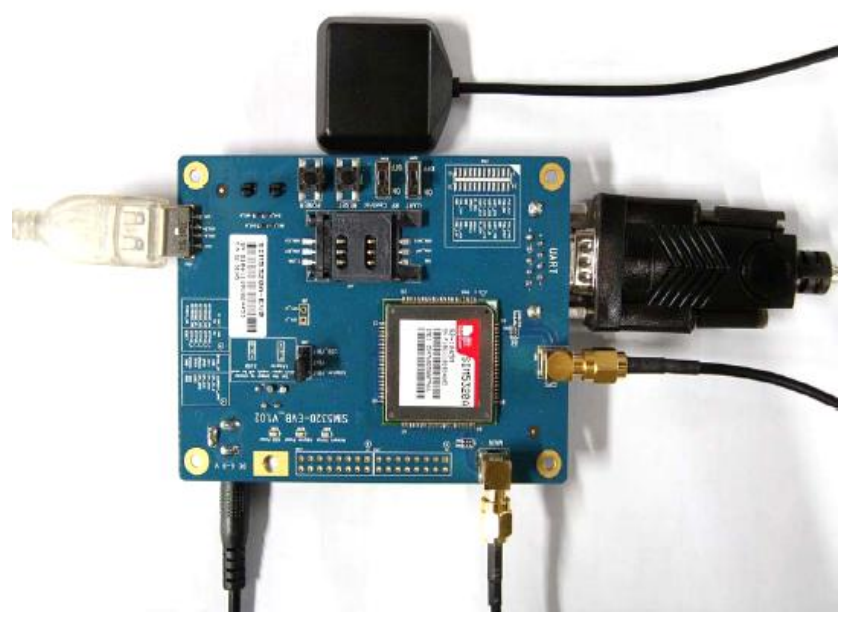

Fig. 4 SIM5230 module and accessories.

\section{B. Alarm Realization}

When a fall is detected, the main control sent AT command to the WCDMA module. The internal GPS will make the accurate positioning and obtain the fall location information. The message with the fall and location information will be sent to a preset telephone number. And the number can set and modify on the device directly. Because the messages are transmitted through WCDMA module, the users can receive the alarm messages normally in any place where there is mobile wireless network.

The GPS functions are provided in AT command format, and they have been designed for us to design GPS applications easily. We can access our GPS AT commands through UART interface which communicates by SIM5230 module.

\section{The System Design and Performance Evaluations}

We have implemented above function and then descript the system designed in details.

\section{A. The Hardware System Design}

The key part of the hardware system is a MCU (microcontroller unit), ADuC7024 form ANALOG DEVICES Inc., which is based on ARM7TDMI core. One three-axis digital accelerometer inertial sensor: LIS331DLH from STMicroelectronics Inc. is being tested. The sensor board is detachable and is connected to the system through I2C interface.

LIS331DLH is an ultra low-power high performance three axes linear accelerometer. It has three dynamically selectable measuring ranges: $\pm 2 \mathrm{~g}, \pm 4 \mathrm{~g}, \pm 8 \mathrm{~g}$, and with the $\pm 2 \mathrm{~g}$ range, it can provide its best sensitivity ( $1 \mathrm{mg} /$ digit). In order to get the maximum resolution and sensitivity, LIS331DLH with $\pm 2 \mathrm{~g}$ is chosen. Besides, there is a low pass filter in LIS331DLH, which can remove some high-frequency noise, and this is also an important reason we chose it.

The firmware executed on the $\mathrm{MCU}$ ADuC7024 is developed in $\mathrm{C}$ language under $\mathrm{uVision} 4 \mathrm{IDE}$. The maximum frequency that the MCU can run is $41.76 \mathrm{MHz}$, however, for the power concern; we set the MCU core clock at $5.22 \mathrm{MHz}$.
The communication between MCU and the three-axis accelerometer is through $\mathrm{I} 2 \mathrm{C}$ bus. The sampling rate of the accelerometer is set to $100 \mathrm{~Hz}$ in our testing and therefore the MCU gets the rectangular coordination $(\mathrm{x}, \mathrm{y}, \mathrm{z})$ of gravity vector from a 3 -axis digital accelerometer every $10 \mathrm{~ms}$. The communication between MCU and the WCDMA module is through UART. The interaction of each module is shown in Fig. 5.
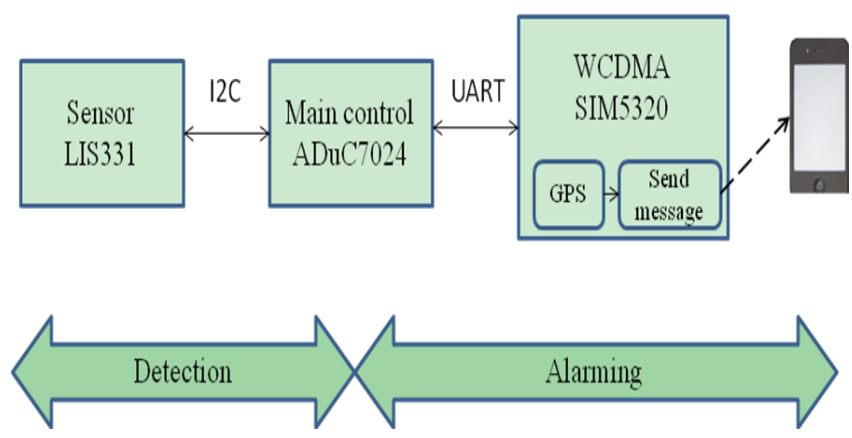

Fig. 5 Block diagram of the system.

\section{B. Results}

In order to verify the practicability of the system, three volunteers have been recruited to test the performance of the system. We first put the sensor device on the experimenter's pocket and set the emergency phone number on it, and then start the system. The fall detecting application is kept running, collecting and recording the reading of the accelerometer sensor. The experimenter performed daily activities such as walking and sitting down and so on while the device has been operated normally. When the experimenter fell down, as shown in Fig. 6, after a few seconds the phone received a message with fall information and location. Every volunteer has finished the test of 40 times and we have gotten the high accuracy rate.

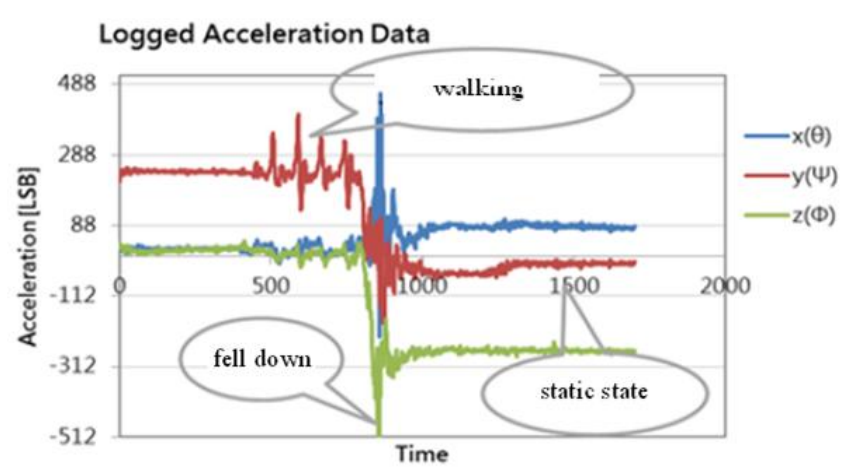

Fig. 6 The result of the fall detection.

\section{Conclusions and Future Work}

A wireless system for fall detection and alarming has been presented, which based on a tri-axis accelerometer controlled by an ARM. The value of $S a$ is used as the judgment standards to detect the fall and the advanced WCDMA technology has 
successfully applied to the system. The experimental results have demonstrated that the proposed system is effective. The system has the advantages of the low cost, high portability and high accuracy rate of fall detecting so that it can be widely used in the elderly health care.

The system will be further expanded, more elderly health monitoring functions will be added, such as gait recording and balanced evaluation, etc. All of the works will have important significance for the health of the elderly people.

\section{References}

[1] Zhenhua Wu and Dasong Deng, "Risk management on social security in China from the perspective of aging population," 2011 International Conference on e-Education, Entertainment and e-Management (ICEEE), pp. 107-109, Dec. 2011.

[2] K. Brewer, C. Ciolek, M. F. Delaune, "Falls in community dwelling older adults: Introduction to the problem," APTA Continuing Education Series, pp. 38-46, July.2007.
[3] Ah-young Jeon, Soo-young Ye, Jun-mo Park, Kwang-nyeon Kim, Jaehyung Kim, Dong-keun Jung, Gye-rok Jeon and Jung-hoon Ro, "Emergency Detection System using PDA based on Self-response Algorithm," 2007 International Conference on Convergence Information Technology, pp.1207 - 1212, Nov. 2007.

[4] Thuy-Trang Nguyen, Myeong-Chan Cho and Tae-Soo Lee, "Automatic fall detection using Wearable Biomedical Signal Measurement Terminal," in Proceedings of 31st Annual International Conference of the IEEE EMBS, pp. 5203 - 5206, Sept. 2009.

[5] Yanbo Tao, Huihuan Qian, Meng Chen, Xin Shi, and Yangsheng Xu, " A Real-time Intelligent Shoe System for Fall Detection," in Proceedings of 2011 IEEE International Conference on Robotics and Biomimetics, pp.2253-2258, December.2011.

[6] Chien-Cheng Lan, Ya-Hsin Hsueh and Rong-Yuan Hu, " Real-Time Fall Detecting System Using a Tri-axial Accelerometer for Home Care," 2012 International Conference on Biomedical Engineering and Biotechnology, pp. 1077 - 1080, May.2012.

[7] Chia-Chi Wang, Chih-Yen Chiang, Po-Yen Lin, Yi-Chieh Chou, I-Ting Kuo, Chih-Ning Huang, and Chia-Tai Chan, " Development of a Fall Detecting System for the Elderly Residents," The 2nd International Conference on Bioinformatics and Biomedical Engineering, 2008. (ICBBE '2008), May. 2008. 